\title{
Erratum: Disorder and superfluid density in overdoped cuprate superconductors [Phys. Rev. B 96, 024501 (2017)]
}

\author{
N. R. Lee-Hone, J. S. Dodge, and D. M. Broun
}

(Received 5 June 2018; published 18 June 2018)

DOI: 10.1103/PhysRevB.97.219903

In Sec. III $\mathrm{C}$ of our paper, the scattering parameters were reported as $\rho_{0} \approx 16 \mu \Omega \mathrm{cm}, \Gamma_{N}=18, \Gamma_{N, \text { Born }}=17$, and $\Gamma_{N \text {,unitarity }}=1 \mathrm{~K}$. The actual values used in the calculations and the preparation of the figures were a factor of $\pi$ larger, i.e., $\rho_{0} \approx 50 \mu \Omega \mathrm{cm}, \Gamma_{N}=18 \pi, \Gamma_{N, \text { Born }}=17 \pi$, and $\Gamma_{N \text {,unitarity }}=\pi \mathrm{K}$. Note that the revised value of $\rho_{0}$ is in good correspondence with the residual terahertz conductivities reported in Fig. S13 of Ref. [1]. In Fig. 4, the plot of gap magnitude $\Delta$ showed the prefactor of the $d$-wave form factor $\cos \left(k_{x} a\right)-\cos \left(k_{y} a\right)$ rather than the gap maximum on the Fermi surface, which is replotted below.

We note that these corrections do not affect the other figures, results, and conclusions of our paper.

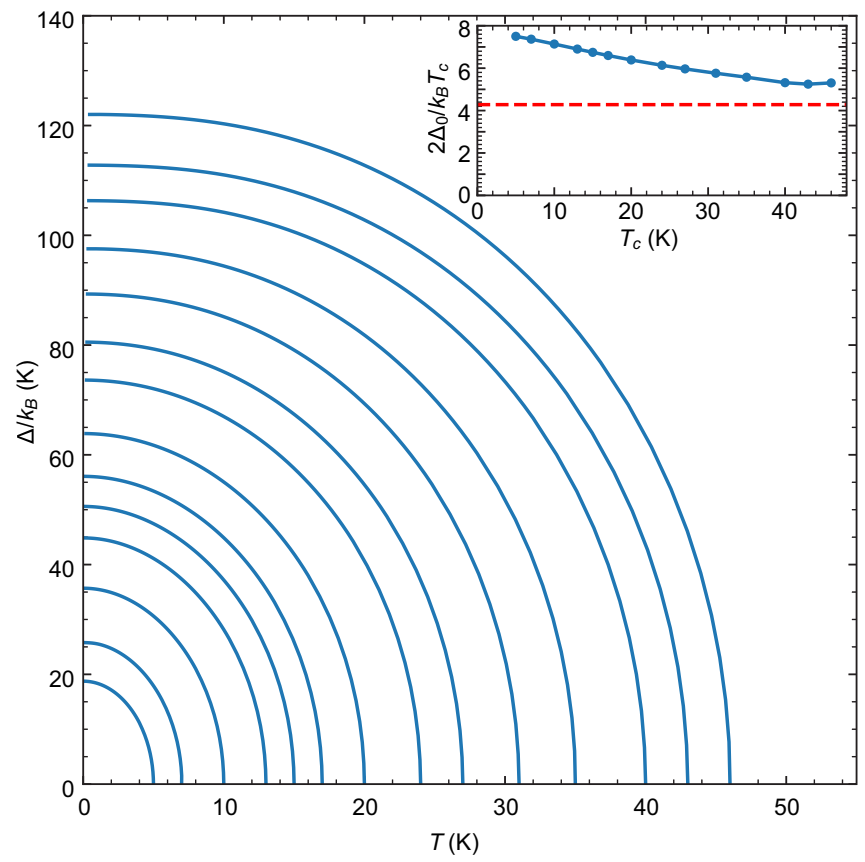

FIG. 4. Revised plot of the gap magnitude $\Delta$, which now correctly shows the $d$-wave gap maximum on the $\mathrm{La}_{2-x} \mathrm{Sr}_{x} \mathrm{CuO}_{4}$ tight-binding Fermi surface. The inset: Revised doping dependence of the zero-temperature gap ratio $2 \Delta_{0} / k_{B} T_{c}$, where $\Delta_{0}$ is now the Fermi-surface gap maximum at zero temperature. The dashed line denotes the clean-limit $d$-wave BCS value for a circular Fermi-surface $2 \Delta_{0}=4.28 k_{B} T_{c}$.

[1] F. Mahmood, X. He, I. Bozovic, and N. P. Armitage, arXiv:1802.02101. 